\title{
A Functional oriT in the Ptw Plasmid of Burkholderia cenocepacia Can Be Recognized by the R388 Relaxase TrwC
}

\section{OPEN ACCESS}

Edited by:

Manuel Espinosa,

Consejo Superior de Investigaciones

Científicas, Spain

Reviewed by:

Fabián Lorenzo,

Universidad de La Laguna, Spain

Radoslaw Pluta,

International Institute of Molecular and

Cell Biology in Warsaw, Poland

*Correspondence:

Matxalen Llosa

Ilosam@unican.es,

Annette C. Vergunst

annette.vergunst@umontpellier.fr

Specialty section

This article was submitted to Molecular Recognition,

a section of the journal

Frontiers in Molecular Biosciences

Received: 11 March 2016

Accepted: 14 April 2016

Published: 03 May 2016

Citation:

Fernández-González E, Bakioui S,

Gomes MC, O'Callaghan D,

Vergunst $A C$, Sangari FJ and Llosa $M$ (2016) A Functional oriT in the Ptw

Plasmid of Burkholderia cenocepacia

Can Be Recognized by the R388 Relaxase TrwC

Front. Mol. Biosci. 3:16.

doi: 10.3389/fmolb.2016.00016

\begin{abstract}
Esther Fernández-González ${ }^{1}$, Sawsane Bakioui ${ }^{2,3}$, Margarida C. Gomes ${ }^{2,3}$, David O’Callaghan ${ }^{2,3}$, Annette C. Vergunst ${ }^{2,3 *}$, Félix J. Sangari ${ }^{1}$ and Matxalen Llosa ${ }^{1 *}$

1 Departamento de Biología Molecular, Instituto de Biomedicina y Biotecnología de Cantabria, Universidad de Cantabria, UC-SODERCAN-Consejo Superior de Investigaciones Cientificas, Santander, Spain, ${ }^{2}$ Institut National de la Santé et de la Recherche Médicale, U1047, Nimes, France, ${ }^{3}$ UFR de Médecine Site de Nimes, U1047, Université de Montpellier, France
\end{abstract}

Burkholderia cenocepacia is both a plant pathogen and the cause of serious opportunistic infections, particularly in cystic fibrosis patients. B. cenocepacia K56-2 harbors a native plasmid named Ptw for its involvement in the Plant Tissue Watersoaking phenotype. Ptw has also been reported to be important for survival in human cells. Interestingly, the presence of PtwC, a homolog of the conjugative relaxase TrwC of plasmid R388, suggests a possible function for Ptw in conjugative DNA transfer. The ptw region includes Type IV Secretion System genes related to those of the F plasmid. However, genes in the adjacent region shared stronger homology with the R388 genes involved in conjugative DNA metabolism. This region included the putative relaxase ptwC, a putative coupling protein and accessory nicking protein, and a DNA segment with high number of inverted repeats and elevated AT content, suggesting a possible oriT. Although we were unable to detect conjugative transfer of the Ptw resident plasmid, we detected conjugal mobilization of a co-resident plasmid containing the ptw region homologous to R388, demonstrating the cloned ptw region contains an oriT. A similar plasmid lacking ptwC could not be mobilized, suggesting that the putative relaxase PtwC must act in cis on its oriT. Remarkably, we also detected mobilization of a plasmid containing the Ptw oriT by the R388 relaxase TrwC, yet we could not detect PtwC-mediated mobilization of an R388 oriT-containing plasmid. Our data unambiguously show that the Ptw plasmid harbors DNA transfer functions, and suggests the Ptw plasmid may play a dual role in horizontal DNA transfer and eukaryotic infection.

Keywords: bacterial conjugation, type IV secretion, Burkholderia cenocepacia, plasmid R388, plasmid Ptw, conjugative relaxases, origin of transfer

\section{INTRODUCTION}

The Burkholderia genus contains more than 60 species of clinical, environmental and agro-biotechnological value (Estrada-De Los Santos et al., 2013); most of them occupy a high diversity of ecological niches, in which they act as plant pathogens and catabolically active soil saprophytes. Only two of them, B. mallei and B. pseudomallei are considered primary pathogens of

Abbreviations: Ptw, plant tissue watersoaking; Dtr, DNA transfer and replication; oriT, origin of transfer; T4SS, Type IV Secretion System. 
animals and humans. The B. cepacia complex $(\mathrm{Bcc})$ is a collection of currently 18 related species, sharing $97.5 \% \mathrm{rDNA}$ sequence similarity, although only $30-60 \%$ genome-wide variability was shown by DNA-DNA hybridization (Vandamme and Dawyndt, 2011). It was initially described as one of the agents that promotes soft rot disease in onions. Importantly, Bcc bacteria pose serious health problems in vulnerable patients, particularly in individuals with cystic fibrosis (CF) or chronic granulomatous disease (CGD), and they are emerging pathogens in nosocomial infections (Coenye and Vandamme, 2003). Members of the Bcc can cause a life-threatening respiratory infection in patients with CF (Gonzalez et al., 1997; Berriatua et al., 2001; LiPuma, 2003). B. cenocepacia and B. multivorans are the most prevalent species $(85-90 \%)$ of the Bcc isolated from patients with $\mathrm{CF}$ (LiPuma, 1998a,b; Speert, 2002). B. cenocepacia is correlated with increased morbidity and mortality, and it has caused several major epidemics (Goven et al., 1993).

The genome of B. cenocepacia strain J2315, isolated from an infected CF patient, has been sequenced (Holden et al., 2009), showing the presence of three circular chromosomes and a plasmid of $92 \mathrm{~kb}$. Most of the coding DNA sequences (CDSs) present in chromosome I are involved in housekeeping functions, while chromosomes II and III [the latter recently renamed as a mega virulence plasmid (Agnoli et al., 2012)] contain CDSs with accessory functions like protective responses and horizontal gene transfer, among others (Holden et al., 2009). Many studies conducted on B. cenocepacia utilize another CF clinical isolate, strain K56-2 (Darling et al., 1998), which is clonally related to J2315 (Mahenthiralingam et al., 2000) but easier to grow and less resistant to antibiotics, making it more amenable to genetic manipulation. K56-2 has a shorter chromosome I due to the absence of a large duplication in J2315, and its $92 \mathrm{~kb}$ plasmid has one less copy of an insertion element (Varga et al., 2013).

Bacterial pathogens use secretion mechanisms for the delivery of virulence determinants (Christie and Vogel, 2000), ranging from one component systems to complex multi-component machineries. Type IV Secretion Systems (T4SS) constitute a family of molecular transporters able to deliver DNA, proteins, or nucleoprotein complexes to the extracellular milieu or other cells (prokaryotic or eukaryotic). Many T4SS mediate secretion of proteins into eukaryotic cells, being implicated in infection processes, and adaptability inside the host (Alvarez-Martinez and Christie, 2009). T4SS involved in conjugative DNA transfer (Zechner et al., 2000) are associated with a DNA transfer region (Dtr), composed by an origin of transfer (oriT), a coupling protein, and a conjugative relaxase; accessory nicking proteins and regulators are also often present. The relaxase catalyzes the initial and final stages of conjugative DNA transfer, cleaving the oriT in the donor to produce the DNA strand to be transferred, and resealing the transferred DNA in the recipient. Relaxases are defined by a series of conserved motifs, and have been classified in different families (Garcillán-Barcia et al., 2009). The oriT is the only DNA requirement in cis for mobilization of a DNA molecule. oriTs are usually located in intergenic regions showing a higher AT content than the rest of the molecule, to allow strand separation during initiation of single-strand transfer. oriT regions usually contain abundance of direct and inverted repeats
(DRs and IRs), many of which have been shown to be the relaxase or accessory nicking protein binding sites (Moncalián et al., 1997; Becker and Meyer, 2000; Williams and Schildbach, 2006, 2007; Lucas et al., 2010; Wong et al., 2011). The Dtr region of plasmid R388 includes a 330 bp oriT and three genes transcribed from a single operon, $\operatorname{trwABC}$ (Llosa et al., 1994). The accessory nicking protein TrwA belongs to the Ribbon-helix-helix (RHH) family proteins (Moncalián and De La Cruz, 2004). TrwB is the coupling protein, interacting both with the transferred substrate and with the Type IV secretion machine (Llosa et al., 2003). The conjugative relaxase TrwC, as other members of the $\mathrm{MOB}_{\mathrm{F}}$ family of relaxases, is composed by two functional domains, the $\mathrm{N}$-terminus harboring the relaxase activity, and the Cterminus showing DNA helicase activity which is also required for conjugation (Llosa et al., 1996).

Two T4SS have been described in B. cenocepacia, the Ptw (Plant tissue watersoaking) T4SS, and the VirB/D4 T4SS (Engledow et al., 2004). The B. cenocepacia VirB/D4 T4SS is located on chromosome II and bears high homology with the prototypical VirB/D4 T4SS of A. tumefaciens. Although its function is still unknown, a possible role in DNA transfer has been reported (Zhang et al., 2009). The Ptw T4SS is encoded on a $45 \mathrm{~kb}$ region (Holden et al., 2009) of a native plasmid of $92 \mathrm{~kb}$. Based on amino acid sequence similarity, it was proposed that the Ptw T4SS is a chimera of various translocation and/or conjugation related proteins similar to VirB/D4 and F-specific subunits (Engledow et al., 2004). One of them, named as PtwC, presents $33 \%$ amino acid identity with $\mathrm{TrwC}$, the relaxase of plasmid R388 (Engledow et al., 2004). The presence of a relaxase homolog, a protein specifically associated with conjugative DNA transfer, argued for a conjugative role of the Ptw T4SS. However, to date Ptw functions have been associated with PtwCindependent secretion of a plant cytotoxic factor (Engledow et al., 2004), and intracellular survival in both professional and non-professional phagocytes (Sajjan et al., 2008).

In this study we address the characterization of the conjugative functions encoded by the $92 \mathrm{~kb}$ plasmid of B. cenocepacia K562 (named pK56-2 by Engledow et al., 2004), hereon referred to as the Ptw plasmid, and the possible relationship with the R388 transfer machinery. We have mapped a functional oriT in Ptw, suggesting that Ptw is a conjugative plasmid; therefore, Ptw may play a dual role in B. cenocepacia, promoting horizontal DNA transfer among bacteria and assisting infection of eukaryotic hosts.

\section{MATERIALS AND METHODS}

\section{Bioinformatic Analysis}

The B. cenocepacia strain J2315 plasmid pBCJ2315 sequence is available in GenBank under the accession number NC_011003. The RAST (Rapid Annotation Subsystems Technology) program was used to re-annotate the ORFs present in the plasmid sequence (Overbeek et al., 2014). PHYRE (Protein Homology/analogy Recognition Engine) was used for the prediction of secondary and 3-dimensional structure of a protein amino acid sequence (Kelley et al., 2015). The phylogenetic tree was constructed using MEGA (Molecular Evolutionary Genetics 
Analysis) software, which employs the Maximum Likelihood method based on the JTT matrix-based model (Koichiro et al., 2011). GenScript bioinformatics software was selected to analyze AT content (http://www.genscript.com). The program Scan for Matches was used to localize IRs having at least $5 \mathrm{bp}$ in length and less than two mismatches (http://www.theseed.org/servers/ downloads/scan_for_matches.tgz). Other intergenic regions of the same size selected randomly from the Ptw plasmid were also analyzed for comparison.

\section{Bacterial Strains and Plasmids}

Bacterial strains used in this study are listed in Table S1. All bacterial strains were grown in Lysogeny Broth (LB), supplemented with $1.5 \%$ agar for culture on solid medium, at $37^{\circ} \mathrm{C}$. Selective media included the following antibiotics at the indicated concentrations: ampicillin (Ap), $100 \mu \mathrm{g} / \mathrm{ml}$; chloramphenicol (Cm), $25 \mu \mathrm{g} / \mathrm{ml}$ (E. coli) or $100 \mu \mathrm{g} / \mathrm{ml}$ (Burkholderia); kanamycin $(\mathrm{Km}), 50 \mu \mathrm{g} / \mathrm{ml}$; nalidixic acid $(\mathrm{Nx})$, $20 \mu \mathrm{g} / \mathrm{ml}$; streptomycin (Sm), $300 \mu \mathrm{g} / \mathrm{ml}$; gentamicin (Gm), 10 $\mu \mathrm{g} / \mathrm{ml}$; and trimethoprim (Tp), $20 \mu \mathrm{g} / \mathrm{ml}$ (E. coli), or $250 \mu \mathrm{g} / \mathrm{ml}$ (Burkholderia). Plasmids are listed in Table 1. Plasmids were maintained in the E. coli lacI ${ }^{\mathrm{q}}$ strain D1210 (Sadler et al., 1980).

\section{Plasmid Constructions}

Plasmids were constructed using standard methodological techniques (Sambrook and Russell, 2001). E. coli DH5 $\alpha$ (Grant et al., 1990) was used in all cloning procedures. Table S2 shows details of the constructions for each plasmid. All cloned DNA inserts were obtained by PCR on genomic DNA isolated from B. cenocepacia K56-2. Genomic DNA was extracted using Instagene Matrix (BioRad). Restriction enzymes, shrimp alkaline phosphatase, and T4 DNA ligase were purchased from Fermentas. High-fidelity Kapa Taq polymerase was purchased from KapaBiosystems. DNA sequences of all cloned PCR segments were determined (MACROGEN Inc. DNA Sequencing Service; Amsterdam, The Netherlands). Plasmid pEF031 was cloned in two sequential steps. In a first step, the 949 bp region where the possible oriT is located was cloned together with the hypothetical accessory nicking protein gene $p t w A$ and 1269 bp of the coupling protein gene $p t w B$, adding a HindIII restriction site in $p t w B$ which did not alter the predicted ORF, obtaining plasmid $\mathrm{pEF} 022$. In a second step, the rest of $p t w B$ and $p t w C$ were added to $\mathrm{pEF} 022$, re-creating the $p t w A B C$ operon.

\section{Colony Analysis by PCR}

All oligonucleotides pairs used for plasmid confirmation by PCR are summarized in Table S3. Colonies obtained from the three plasmid curing methods were checked for the presence of the Ptw plasmid by PCR, using two oligonucleotide pairs (P1-P2) and (P3-P4) amplifying $p t w C$ and $p w a C$ internal fragments. All the colonies tested, at least 50 for each curing method, were positive for the presence of both genes. Four oligonucleotide pairs (P5-P6; P7-P8; P9-P10; and P11-P12) were selected to detect the presence of different regions of the $45 \mathrm{~kb}$ ptw cluster in the two B. cepacia strains (Table S1) by PCR. K56-2 total DNA was always used as a positive control. Transconjugants obtained in the mobilization assay were checked by PCR using oligonucleotide pairs (P13P14) to amplify the R388 oriT sequence of plasmid pSU1445, and (P15-P6) pair to confirm the presence of the cat gene in the pBBR1 plasmid.

\section{Plasposon Insertion}

For preparation of electrocompetent cells, bacteria were grown to $\mathrm{OD}_{600}=0.5-0.6$, and pelleted by centrifugation at $4^{\circ} \mathrm{C}$. Two series of washes and centrifugations (6000 rpm) of 1 vol milliQ water and a final wash in $1 / 50$ vol $10 \%$ glycerol at $4^{\circ} \mathrm{C}$ were applied. Cells were resuspended in 1/500 vol $10 \%$ glycerol and aliquotted in $50 \mu \mathrm{l}$ samples. K56-2 electrocompetent cells were transformed with $<10 \mathrm{ng}$ of the pTnMob-OCm plasposon DNA (Dennis and Zylstra, 1998) in a $0.2 \mathrm{~cm}$ Gene Pulser ${ }^{\circledR}$ cuvette (BioRad) and subjected to an electric pulse $(2.5 \mathrm{kV}, 25 \mathrm{Mf}$, and $200 \Omega$ ) in a MicroPulser TM (BioRad). Electroporated cells were added to $1 \mathrm{ml} \mathrm{LB}$ and incubated with shaking at $37^{\circ} \mathrm{C}$ to allow expression of antibiotic resistance genes. After incubation cells were plated on antibiotic containing media. The pool of bacterial colonies growing on $\mathrm{Cm}$ plates was used as donor cells to test Ptw conjugation. Considering the size of the plasmid and the total length of the genomic DNA, it is estimated that on average one out of 100 colonies would have the plasposon inserted in the Ptw plasmid, and from those, about half would not affect

TABLE 1 | Plasmids used in this work.

\begin{tabular}{|c|c|c|c|}
\hline Plasmid & Antibiotic Resistance & Description & References \\
\hline pBBR1-MCS & $\mathrm{Cm}^{\mathrm{R}}$ & Broad host range vector from Bordetella bronchiseptica & Kovach et al., 1994 \\
\hline pEF022 & $\mathrm{Cm}^{\mathrm{R}}$ & pBBR1::Ptw-oriT +ptwA+2/3ptwB & This work \\
\hline pEF033 & $\mathrm{Cm}^{\mathrm{R}}$ & pBBR1::Ptw-oriT (700 bp) & This work \\
\hline pEF034 & $\mathrm{Cm}^{\mathrm{R}}$ & pBBR1::Ptw-oriT (700 bp)+ptwA & This work \\
\hline pSU1443 & $\mathrm{Km}^{\mathrm{R}} \mathrm{Tp}^{\mathrm{R}}$ & R388::Tn5tac in trwB & Llosa et al., 1994 \\
\hline pSU1445 & $\mathrm{Km}^{\mathrm{R}} \mathrm{Tp}^{\mathrm{R}}$ & R388::Tn5tac in trwC & Llosa et al., 1994 \\
\hline pSU2007 & $\mathrm{Km}^{\mathrm{R}}$ & R388 with a $\mathrm{Km}^{\mathrm{R}}$ cassette & Martinez and de la Cruz, 1988 \\
\hline pTnMob-OCm & $\mathrm{Cm}^{\mathrm{R}}$ & Self-cloning minitransposon & Dennis and Zylstra, 1998 \\
\hline
\end{tabular}


any transfer-related functions. Therefore, in a mating experiment involving $10^{6}$ donor cells, we would expected to have at least $5 \times 10^{3}$ cells carrying a $\mathrm{Cm}$-resistant, transfer-proficient $\mathrm{Ptw}$ plasmid.

\section{Mating Experiments}

Standard E. coli quantitative mating assays were performed as described previously (Grandoso et al., 2000): equal amounts of donor and recipient strains from overnight cultures were mixed and placed on Millipore filters on a prewarmed LB agar plate for $1 \mathrm{~h}$ at $37^{\circ} \mathrm{C}$. Strains D1210 and DH5 $\alpha$ were used as donors and recipients, as indicated. Results are shown as the frequency of transconjugants per donor and are the mean of 3-5 independent experiments. For mating assays using $B$. cenocepacia as a donor and E. coli $\beta 2163$ (Demarre et al., 2005) or B. cepacia strains as recipients, bacterial cultures were adjusted to an $\mathrm{OD} 600=0.5$, mixed at an equal ratio, washed twice and transferred to a Millipore filter on a prewarmed LB agar plate for $18 \mathrm{~h}$ at $37^{\circ} \mathrm{C}$.

\section{Plant Watersoaking Assay}

The plant watersoaking assay was used to assess the functionality of the Ptw T4SS in B. cenocepacia, as described (Engledow et al., 2004). Several onion types, like echalote, red onion and white onion were used throughout the study, and no significant differences were found among them, so all experiments were performed with white onions. Bacterial suspensions of B. cenocepacia strains were adjusted to $\mathrm{OD}_{600}=0.5$ and individual onion scales were wounded on the abaxial (inner) surface with a sterile blade. $10 \mu \mathrm{l}$ of bacterial suspension $\left(10^{6}\right.$ c.f.u per scale) were inoculated into the wound. Sterile double-distilled water was used as a negative control. Onion scales were placed on a sheet of sterile aluminum foil in containers containing Whatman paper towels moistened with sterile distilled water, sealed, and incubated at $37^{\circ} \mathrm{C}$. Ptw activity was estimated by the appearance of water drops on the onion tissue at $24 \mathrm{hpi}$.

\section{Plasmid Curing}

In order to cure the Ptw plasmid from B. cenocepacia, bacteria were incubated under different stress conditions, where mutations in essential plasmid genes can be induced, promoting the loss of the plasmid. Two methods using high temperatures and a third one growing bacteria in the presence of the mutagenic agent ethidium bromide were used: (i) Growth on TN Medium (Gonzalez et al., 1997). Bacteria containing the plasmid to be cured were grown in TN broth [5 $\mathrm{g}$ Tryptone, $1 \mathrm{~g}$ Dextrose (D-Glucose), $2.5 \mathrm{~g}$ Yeast extract, and $8.5 \mathrm{~g} \mathrm{NaCl}$ per litter] for 18 $\mathrm{h}$ at $37^{\circ} \mathrm{C}$ with shaking (200 r.p.m). Bacteria were subcultured into pre-warmed TN broth $\left(42-44^{\circ} \mathrm{C}\right)$ to a final concentration of $10^{4}$ c.f.u ml $\mathrm{m}^{-1}$ and grown with shaking in a water bath (at $42-44^{\circ} \mathrm{C}$ ) for $18 \mathrm{~h}$. Temperature-treated cultures were diluted and plate on TN agar. Individual colonies were checked by PCR. (ii) Growth at High Temperature (Asheshov, 1966). Bacteria were grown overnight at $37^{\circ} \mathrm{C}$. Overnight cultures were diluted $1 / 4$ in fresh LB medium for $11 / 2 \mathrm{~h}$. Samples of this culture were added to tubes containing $5 \mathrm{ml}$ of fresh $\mathrm{LB}$ medium previously pre-warmed at $37^{\circ} \mathrm{C}$ and at $43-44^{\circ} \mathrm{C}$ respectively. Tubes were then incubated at the appropriate temperature for
$51 / 2 \mathrm{~h}$. Cultures were then diluted and spread on LB agar plates and incubated at $37^{\circ} \mathrm{C}$ overnight; the resulting colonies were then tested for the loss of the native plasmid by PCR. (iii) Growth with Ethidium Bromide (Crameri et al., 1986). Ethidium bromide $(25 \mu \mathrm{g} / \mathrm{ml})$ was added to a B. cenocepacia liquid culture and incubated with shaking at $37^{\circ} \mathrm{C}$ overnight protected from the light. A 1/10 dilution of the overnight culture was prepared with fresh LB medium supplemented with Ethidium bromide. This procedure was repeated over 15 days; every 2 days, dilutions of the culture were plated, and colonies were checked for the presence of the plasmid by PCR.

\section{RESULTS}

\section{Bioinformatic Analysis of the Ptw Plasmid}

A preliminary annotation of the Ptw plasmid sequence for B. cenocepacia J2315 was available (Holden et al., 2009). We have used the RAST Bioinformatic Program to obtain a detailed annotation of the ORFs present in the plasmid, based on a comparative study against the NCBI database. As previously reported (Engledow et al., 2004; Holden et al., 2009), a $45 \mathrm{~kb}$ region was found to encode genes with similarity to the F plasmid T4SS genes (Lawley et al., 2003). However, the adjacent DNA region to T4SS genes, which included the putative relaxase and coupling protein genes previously described, $p t w C$ and $p t w D 4$, showed higher similarity to the Dtr region of conjugative plasmid R388: PtwC shows $33 \%$ of amino acid identity with R388 relaxase TrwC, and 27\% with relaxase TraI of the F plasmid. PtwD4 presents 20\% identity with VirD4 from A. tumefaciens, 28\% with TraD of the F plasmid, and 30\% with R388 TrwB. Due to the stronger similarity to TrwB, PtwD4 will be named PtwB from here on.

Both R388-TrwC and F-TraI belong to the $\mathrm{MOB}_{\mathrm{F}}$ family of relaxases, defined by three common motifs based on the known atomic structure of the TrwC relaxase and similar proteins (Francia et al., 2004). CLUSTALW alignment of PtwC and its homologs showed that the three relaxase motifs were conserved in PtwC (Figure 1A). The homology between TrwC and PtwC in this amino terminal domain goes up to $42 \%$, while the Cterminal domains share $29 \%$ amino acid identity. In addition, PtwC shares the seven conserved amino acid motifs in the Cterminal domain which define the DNA helicase superfamily I (Matson, 1990; Figure 1A). The MEGA5 program (Koichiro et al., 2011) was used to determine its phylogenetic position in $\mathrm{MOB}_{\mathrm{F}}$ family (Figure S1), confirming that PtwC belongs to the $\mathrm{MOB}_{\mathrm{F}}$ family, and R388-TrwC is its closest relative out of the Burkholderia spp homologs.

In most conjugative systems, the oriT is located close to the relaxase; accessory nicking proteins are also often encoded in this DNA region. Analysis of the DNA region upstream of $p t w B$ led to the identification of a small ORF of $429 \mathrm{bp}$ (pBCA060) coding for a hypothetical protein with a predicted $\mathrm{RHH}$ structure related to other members of the RHH superfamily (Heidelberg et al., 2000), and thus a likely accessory nicking protein of PtwC; we named it PtwA after TrwA, the accessory nicking protein of TrwC, although both proteins do not share significant amino acid 
A

\section{RELAXASE MOTIFS}

\section{MOTIF I}

MOTIF II

MOTIF III

TrWC (1) MLSHMVLTRQDIGRAASYYEDGADDYYAKD 〈50> IGLDLTFSAPKSVSLQA 〈45> VIGKFRHETSRERDPQLHTHAVI (166)

TraI_R46 (1) MLDITTITRQNVTSVVGYYSDAKDDYYSKD <49> LGYDLTFSAPKGVSMQA 〈45> VVATFRHETSRALDPDLHTHAFV (165)

PtwC $^{-}$(1) MLSLHNIA--SAAQTRNYFS--KDNYYTQD $\langle 56\rangle$ PGIDITFSAPKSVSLMA $\langle 50\rangle$ VAALFKHNTSRDLDPQTHTHAVV (169)

TraI_F (1) MMSIAQVR--SAGSAGNYYTD-KDNYYVLG <49> PGYDLTFSAPKSVSMMA <45> VMALFNHDTSRDQEPQLHTHAVV (162)

\section{HELICASE MOTIFS}

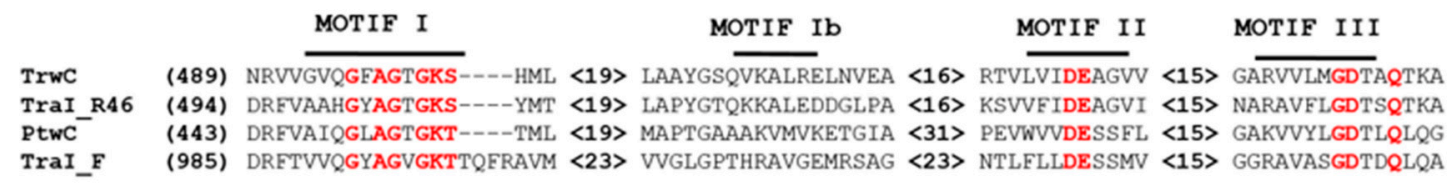

\begin{tabular}{|c|c|c|c|c|c|c|c|}
\hline & & MOTIF IV & & MOTIF V & & MOTIF VI & \\
\hline $\mathrm{wc}$ & (606) & DQLQAAGMQT & $\langle 224\rangle$ & DHAYATTVHSSQGLTSDRVLIDAH & $\langle 9\rangle$ & VYYVAISRARFEAR & (892) \\
\hline TraI_R46 & (611) & EQLIKAGMQT & $\langle 230\rangle$ & SYAYATTVHKSQGLTCDRVLFNID & $\langle 9\rangle$ & VFYVGISRARHEVE & (896) \\
\hline 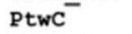 & (575) & ELAQKDGIAT & $\langle 260\rangle$ & DHAYASTVHAAQGASRNTTIFAIN & $\langle 24\rangle$ & SYYVGVTRASHDIS & (897) \\
\hline $\mathbf{F}$ & (1113) & LQQTRSAADV & $\langle 281\rangle$ & DLAYAITAHGAQGASETFAIALEG & $\langle 12\rangle$ & SAYVALSRMKQHVQ & (1444) \\
\hline
\end{tabular}

B

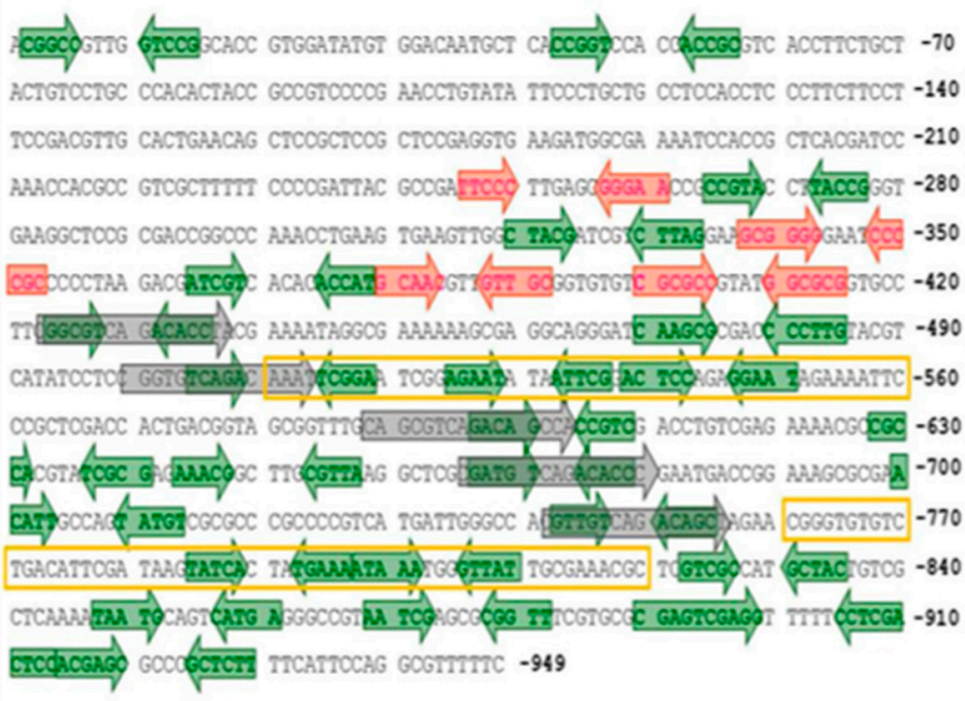

FIGURE 1 | Conserved motifs in PtwC and related proteins and features of the hypothetical oriT region. (A) Conserved motifs in the relaxase and DNA helicase domains, with the most conserved residues highlighted in red. (B) Features in hypothetical Ptw oriT region (coordinates 66.438-65.490 in Accession number NC_011003.1). IRs with one mismatch are represented in green. A set of DRs of 15 bp with three mismatches are represented in gray. High AT regions are marked with a yellow box.

identity. The most likely region to contain the Ptw oriT sequence would be in a $949 \mathrm{bp}$ intergenic region located upstream of $p t w A$. It contains DNA segments with high AT content (squared regions in Figure 1B), candidates to contain the nic site, and two times more IRs than in a randomly selected intergenic region of the same size (not shown), especially in the region including the two AT-rich DNA segments (Figure 1B). All these features lead us to propose this as a candidate region to contain the Ptw oriT.

Compiling the results of the bioinformatics analysis, we propose that the Ptw plasmid codes for a conjugative transfer region with a T4SS related to that of the F plasmid, while the
Dtr region is closely related to that of the R388 transfer system (Figure 2).

\section{Involvement of the Ptw Plasmid in Conjugative DNA Transfer}

Our next goal was to experimentally test conjugative transfer of the Ptw plasmid. Entry exclusion is a common phenomenon in conjugative DNA transfer, which prevents conjugation into a recipient cell already containing the conjugative plasmid present in the donor (Garcillán-Barcia and de la Cruz, 2008). To prevent this possible effect, a Ptw-free recipient strain was required. We 
A F-plasmid

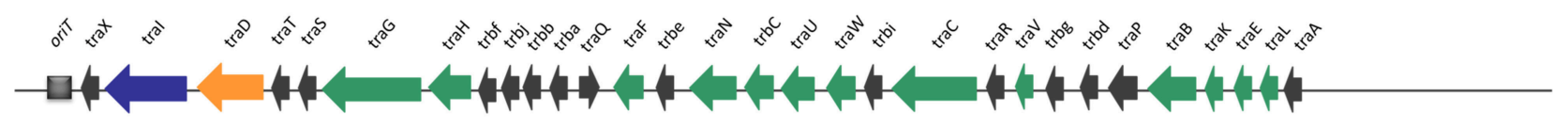

B Ptw-plasmid

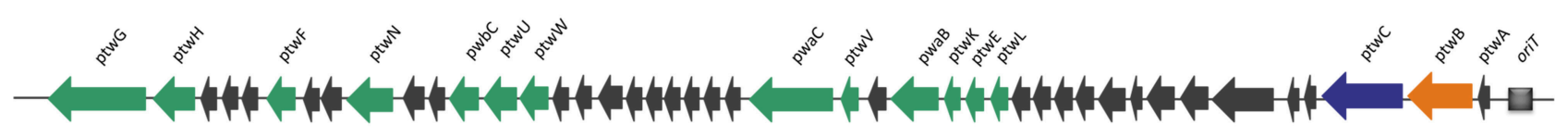

C R388-plasmid

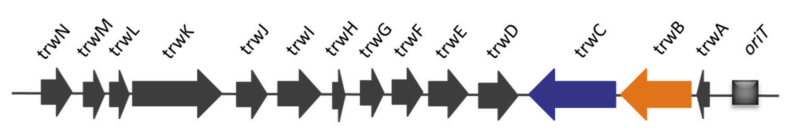

FIGURE 2 | Comparison of DNA transfer regions of conjugative plasmids (A) F, (B) Ptw, and (C) R388, highlighting structural and sequence homologies. Homologous ORFs are highlighted in a color-coded format: T4SS genes are shown in green, relaxases in blue, and coupling proteins in orange. Ptw genes are named based on their closest homologs in $\mathrm{F}$ and R388 plasmids.

TABLE 2 | Mating assays to test mobilization of the Ptw Dtr region.

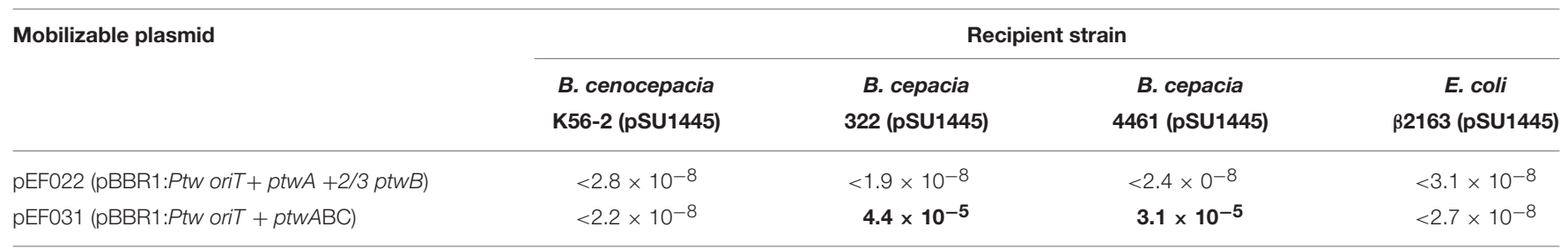

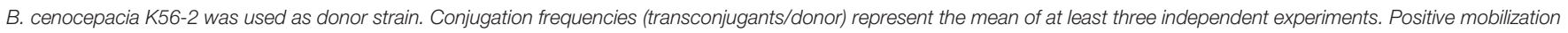
frequencies are highlighted in bold.

attempted to cure a B. cenocepacia strain of the plasmid by three different methods (see Materials and Methods); colonies were checked by PCR and assayed for ability to induce the plant watersoaking phenotype; not a single colony out of about 150 screened colonies (50 from each method) had lost the plasmid.

The Ptw plasmid has been described only in B. cenocepacia (Engledow et al., 2004). Therefore, other bacterial species were selected to be used as recipients in the conjugation assays (Table S1). No Ptw homologs are present in the five available B. cepacia genomes, so we used two ATCC B. cepacia strains as recipients. Since their genomic sequence is not available, we checked for the presence of identical or highly homologous $p t w$ sequences by PCR amplification of four different regions of the $45 \mathrm{~kb} p t w$ cluster, which were all negative. To detect Ptw plasmid transfer, we inserted a p $\mathrm{TnMob}$-Cm plasposon by electroporation in K562. Colonies resistant to $\mathrm{Cm}$ were used as donors in conjugation to K56-2, B. cepacia 322 and E. coli $\beta 2133$. After a series of transfer experiments using about $5 \times 10^{9} \mathrm{Cm}$-resistant donor colonies, transconjugants were never obtained. The absence of Ptw DNA transfer could be due to natural repression or lack of induction of this transfer system.

It cannot be discarded that transconjugants were not detected in B. cepacia due to lack of replication of the Ptw plasmid, or incompatibility with some resident plasmid. These limitations would be overcome by using a different mobilizable replicon. The putative Ptw Dtr region, consisting of $p t w A B C$ and oriT, was cloned on the broad-host-range pBBR1 replicon (Kovach et al., 1994), and a plasmid carrying the Ptw oriT but without the putative relaxase $p t w C$ was also constructed. The plasmids were introduced in B. cenocepacia K56-2, which naturally contains the Ptw plasmid, providing the $p t w$ transfer genes also in trans. Recipient strains harboring a trimethoprim resistant plasmid (pSU1445; Table 1) were used to allow selection of transconjugants. Mating assays were carried out as described in Experimental Procedures, and results are shown in Table 2. When assaying mobilization of a plasmid carrying the putative Ptw oriT, $p t w A$, and a truncated copy of $p t w B$, no transconjugants were ever obtained, in spite of the presence of 
the Ptw plasmid which provides the complete putative transfer machinery. However, a pBBR-plasmid containing the complete Ptw Dtr region was mobilized to both $B$. cepacia strains; no mobilization to B. cenocepacia K56-2 or to E. coli was detected. Transconjugants were verified by PCR amplification to confirm the presence of both plasmid pSU1445 and the mobilized plasmid. This result proves the presence of a functional oriT in the Ptw plasmid.

\section{Recognition of the Ptw oriT by the Conjugative Relaxase TrwC}

It has previously been reported that conjugative systems can use heterologous T4SS with different affinities: the TrwCDNA complex could be secreted through the T4SS of different conjugative plasmids, and moreover, it could also use the T4SS of Bartonella henselae to be transferred to human cells (Llosa et al., 2003; Fernández-González et al., 2011). Mobilization of a plasmid coding for the Ptw Dtr components by other conjugative T4SS was therefore tested. Plasmids containing different parts of the Ptw Dtr were tested for mobilization between E. coli strains. Three different conjugative plasmids were used as helper plasmids in the donor strain: pSU2007 (R388 derivate) (Martinez and de la Cruz, 1988), pOX38-Km (F derivative) (Chandler and Galas, 1983), and pKM101 (R46 derivative; Langer and Walker, 1981; Table 1), all coding for relaxases sharing homology with PtwC (Figure 1). Results are shown in Table 3. While no transconjugants were observed with the F or R46 transfer systems, a low level of mobilization of the Ptw-oriT containing plasmids was observed when the R388 transfer system was present in the donor cell. Transconjugants were analyzed by restriction analysis (data not shown), showing both the mobilizable and helper plasmids as separate entities in the transconjugants (clearly distinguishable by their different copy number), thus ruling out plasmid conduction by cointegrate formation with the conjugative plasmid. Restriction analysis with HindIII and SacI rendered the expected fragments for the helper pSU2007 $(32.5 \mathrm{~kb})$ and mobilizable plasmids pEF022 or pEF031: $2640 \mathrm{bp}$ fragment in all cases ( $p t w$ oriT $+p t w A+2 / 3 p t w B$ ) plus a fragment of 4700 or $8800 \mathrm{bp}$, respectively.

Surprisingly, similar results were obtained when the mobilizable plasmid carried only the Ptw oriT, ptwA, and $2 / 3$ of $p t w B$, instead of the complete Ptw Dtr region (Table 3, compare mobilization by pSU2007 of pEF022 and pEF031). Thus, mobilization through the T4SS of R388 is not due to PtwC, but presumably to recognition of the Ptw oriT by TrwC in trans. To confirm if mobilization is TrwC dependent, a TrwC-deficient R388 plasmid (pSU1445) was used as helper; in this case, no plasmid mobilization was detected (Table 3). These assays also show that there is no functional complementation of TrwB or TrwC by PtwB and PtwC, at least in trans, since R388 trwB(pSU1443) and R388 trwC- (pSU1445) deficient plasmids cannot be mobilized in the presence of a plasmid harboring the Ptw

TABLE 3 | Mobilization of plasmids carrying the Ptw-oriT by different conjugative plasmids between E. coli strains.

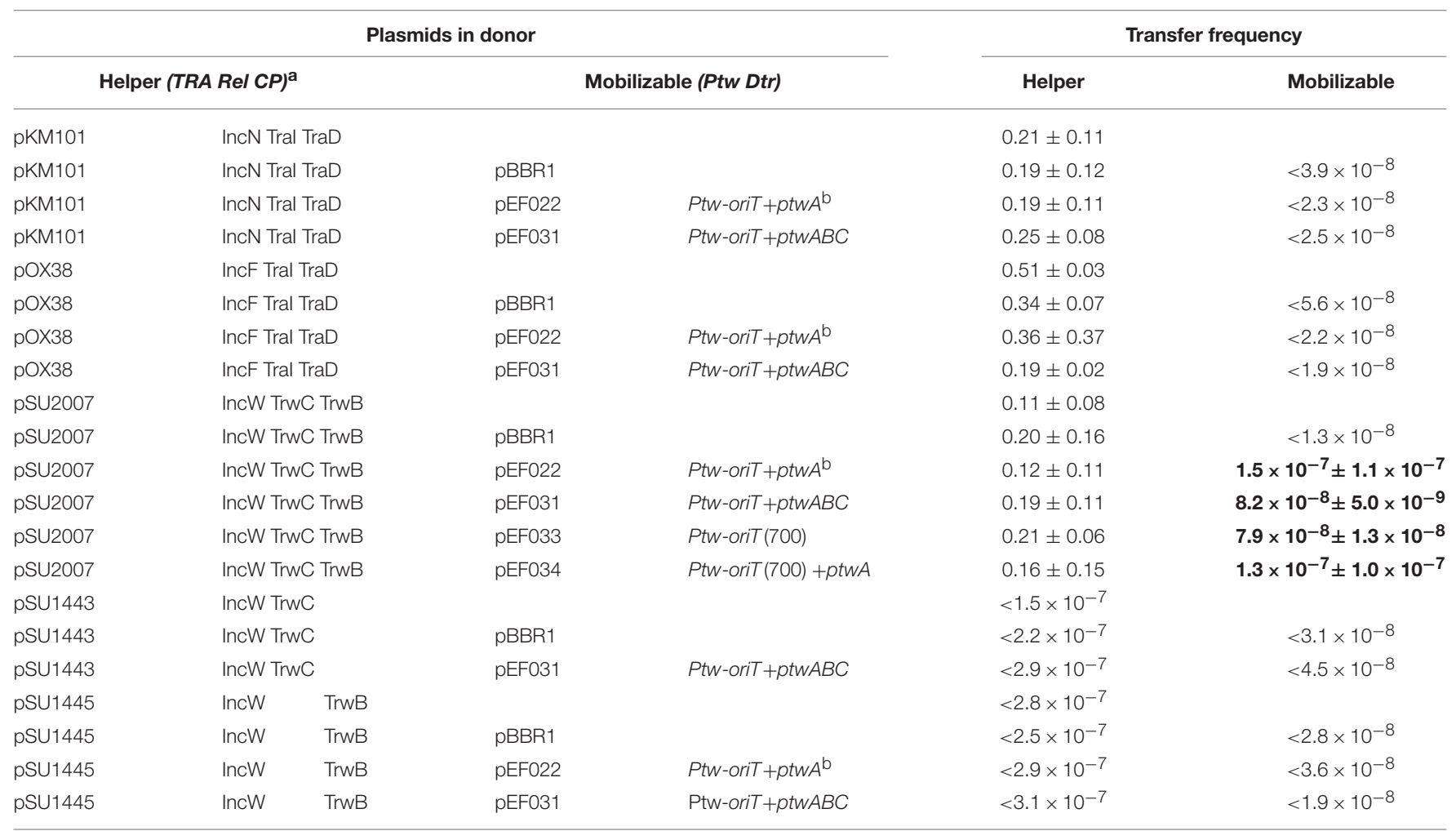

DH5 $\alpha$ was used as a donor and D1210 as a recipient. Transfer frequencies (transconjugants/donor) represent the mean \pm SD of three independent experiments. ${ }^{a}$ Description of the Helper plasmid: TRA transfer system (defined by plasmid Inc group), Rel relaxase, CP coupling protein.

${ }^{b} A$ truncated ptwB copy was also present, which was omitted for clarity. Positive mobilization frequencies are highlighted in bold. 
Dtr region (Table 3, negative transfer frequency of pSU1443 and pSU1445 in the presence of pEF031).

Mobilization of Ptw-oriT containing plasmids by R388 allowed us to refine mapping of the Ptw oriT. Plasmids were constructed carrying only the region where the oriT-like features were found, with and without $p t w A$. A plasmid carrying region 65.490-66.190 of the Ptw-oriT (nucleotides 249-949 in Figure 1B) was mobilized by TrwC as efficiently as the plasmid containing the complete Ptw Dtr region (Table 3, compare mobilization frequency of pEF031 and pEF033 by pSU2007).

\section{DISCUSSION}

Previous studies proposed that the T4SS encoded on the Ptw plasmid of B. cenocepacia played a role in interactions with the eukaryotic host. The T4SS was associated with the plant tissue watersoaking phenotype, produced by unknown translocated effector proteins (Engledow et al., 2004). In addition, the Ptw T4SS was shown to have a role in intracellular survival and replication of $B$. cenocepacia inside macrophages (Sajjan et al., 2008), although Valvano and colleagues were unable to show defects in intracellular survival in B. cenocepacia mutants lacking all possible secretion systems (Valvano, 2015). However, the presence of the $p t w C$ gene coding for a putative conjugative relaxase, not involved in the Ptw phenotype (Engledow et al., 2004), led us to hypothesize that Ptw is a conjugative plasmid. Relaxases are the signature proteins of conjugative systems; all known close homologs are involved in horizontal DNA transfer (Garcillán-Barcia et al., 2009). PtwC belongs to the $\mathrm{MOB}_{\mathrm{F}}$ family (Garcillán-Barcia et al., 2009) of relaxases-helicases, according to phylogenetic analysis (Figure S1). Conservation in PtwC of the signature motifs associated to relaxase and DNA helicase activity (Figure 1) reinforce the idea that $\mathrm{PtwC}$ is an active conjugative relaxase.

A straightforward way to determine the role of the Ptw plasmid would be to cure it from B. cepacia strains; however our repeated attempts were unsuccessful. Low-copy-number plasmids have developed a number of mechanisms to promote their stable maintenance, such as Toxin-Antitoxin (TA) systems (Yamaguchi et al., 2011). The Ptw plasmid indeed encodes genes showing similarity to the toxin and antitoxin of the VapBC TA system of Mycobacterium tuberculosis (Ramage et al., 2009), which could explain the failure to cure the Ptw plasmid. Similarly, recent studies have shown that the pC3 mega-plasmid (previously chromosome 3 ) in B. cenocepacia K56-2 showed unexpected stability due to the presence of a toxin-antitoxin system, which made it difficult to cure this non-essential plasmid from K56-2, in contrast to other Bcc strains (Agnoli et al., 2012).

The Ptw T4SS was reported to be a chimera between VirB and F-like specific subunits (Engledow et al., 2004). Our bioinformatic analyses show that the plasmid possesses all the components of a conjugative system, composed of a T4SS gene set related to that of the F plasmid, and a Dtr region whose closest homolog is that of plasmid R388 (Figure 2). In the Dtr region, upstream the putative coupling protein and relaxase previously reported (Engledow et al., 2004), we described a protein, PtwA, with similar size to accessory nicking proteins, predicted to have the characteristic Ribbon-Helix-Helix secondary structure. More importantly, we delimited a region upstream of this putative accessory nicking protein candidate to be the Ptw oriT, due to its high AT content and the elevated number of IRs and DRs (Figure 1).

Despite the presence of a DNA transfer region, Ptw plasmid conjugation could not be detected under different test conditions. However, we detected mobilization of a Ptw-Dtr containing plasmid introduced in the host of the Ptw plasmid. It is reasonable to assume that mobilization will occur through the Ptw T4SS, although we cannot discard that the plasmid may be using the VirB T4SS, previously shown to be involved in DNA transfer (Zhang et al., 2009). Whatever the T4SS used for secretion, the absence of mobilization of the Ptw plasmid, which includes the Dtr region, suggests that self-transfer is repressed in cis. This is also the case for one of the symbiotic plasmids (pSym) present in Rhizobium etli, which possesses a full set of genes involved in conjugation. Plasmid transfer has never been detected under laboratory conditions, although there are several lines of evidence for the movement of pSym plasmids among naturally occurring rhizobial populations (Wernegreen and Riley, 1999). The absence of conjugation in this symbiotic plasmid has been explained by the presence of a conjugative repressor, $r c t A$, which inhibits the conjugation of the symbiotic plasmid by decreasing virB transcription (Sepúlveda et al., 2008). No similar genes to $r c t A$ have been described until now in the Ptw plasmid, however the presence of such a repressor cannot be discarded.

Mobilization of the plasmid containing the Ptw Dtr occurred to two different B. cepacia strains, but not to B. cenocepacia or to E. coli (Table 2). The absence of transfer to B. cenocepacia could reflect an entry-exclusion phenomenon, frequent in bacterial conjugative plasmids (Garcillán-Barcia and de la Cruz, 2008), due to the presence of the Ptw plasmid in the recipient. The absence of transfer to $E$. coli could reflect a narrow host range of this conjugative system, similar to that of the F plasmid to which the T4SS is most closely related (Zhong et al., 2005).

Plasmids containing the putative oriT but no $p t w C$ were not mobilized (Table 2), supporting the sequence homology and phylogenetic analysis which indicate that $\mathrm{PtwC}$ is a conjugative relaxase. Since, $p t w C$ is present in the co-resident Ptw plasmid and no Ptw transfer is detected, PtwC may act only in cis, as previously reported for other conjugative relaxases (PérezMendoza et al., 2006; Cho and Winans, 2007). Alternatively, this could be due to cis-repression of the ptwC copy present in the Ptw plasmid, as discussed above. The $p t w C$ copy present in the multicopy plasmid would escape repression in trans, or if the presence of multiple $p t w C$ copies outnumbers the repressor, leading to partial expression (in accordance with the low transfer frequencies obtained).

Interestingly, we showed that the Ptw oriT can be also recognized by the conjugative relaxase of plasmid $\mathrm{R} 388$, TrwC (Table 3), while the reverse is not true: PtwC does not act on R388 oriT, at least in trans. The fact that TrwC can mobilize Ptw-oriT containing plasmids in trans while PtwC cannot, can be explained by the different nature of both relaxases. cis- or trans-acting is an attribute of the relaxase, not the oriT. PtwC may act in cis as has been reported for a few other relaxases, but most of them act in trans, including TrwC (Llosa et al., 1996). If alternatively the lack of trans-mobilization by $\mathrm{PtwC}$ were due to cis-repression of the 
$p t w C$ gene, as suggested above, this repression would obviously not affect the trwC copy present in a different plasmid.

Traditionally considered as highly sequence specific, conjugative relaxases have been shown to act on related sequences with relaxed specificity (Jandle and Meyer, 2006; Fernández-López et al., 2013; O’Brien et al., 2015; Pollet et al., 2016). However, it is surprising that TrwC can act on another oriT with no apparent homology to its natural target; we have not found any sequence within the $700 \mathrm{bp}$ defined as the Ptw oriT which resembles the R388 nic site and the surrounding 17 bp which are required for TrwC binding and nicking (Lucas et al., 2010). TrwC has been reported to recognize sequences which differ from its natural target (Agúndez et al., 2012), and it is also efficiently recruited by heterologous T4SS (Llosa et al., 2003; Fernández-González et al., 2011), so this result adds to previous ones to highlight the promiscuity of this enzyme and its conjugative transfer system. In addition, this result opens up the possibility that the Ptw plasmid can be transferred in nature by the conjugative system of a horizontally transmitted promiscuous plasmid such as is R388.

Earlier reports have suggested a role for the Ptw T4SS in the Plant Tissue Water soaking phenotype and survival in mammalian host cells through the secretion of putative effector proteins (Sajjan et al., 2008). Our results unambiguously show that the Ptw plasmid encodes an active oriT adjacent to the ptw T4SS gene cluster. It would be interesting to find out whether conjugative transfer during infection of mammalian hosts would contribute to disease. A role for the Ptw plasmid in transfer of DNA and effector proteins may give an advantage to $B$. cenocepacia in the environment, while at the same time contributing to their opportunistic character.

\section{REFERENCES}

Agnoli, K., Schwager, S., Uehlinger, S., Vergunst, A., Viteri, D. F., Nguyen, D. T., et al. (2012). Exposing the third chromosome of Burkholderia cepacia complex strains as a virulence plasmid. Mol. Microbiol. 83, 362-378. doi: 10.1111/j.1365-2958.2011.07937.x

Agúndez, L., González-Prieto, C., Machón, C., and Llosa, M. (2012). Sitespecific integration of foreign dna into minimal bacterial and human target sequences mediated by a conjugative relaxase. PLoS ONE 7:e31047. doi: 10.1371/journal.pone.0031047

Alvarez-Martinez, C. E., and Christie, P. J. (2009). Biological diversity of prokaryotic type IV secretion systems. Microbiol. Mol. Biol. Rev. 73, 775-808. doi: 10.1128/MMBR.00023-09

Asheshov, E. H. (1966). Loss of antibiotic resistance in Staphylococcus aureus resulting from growth at high temperature. J. Gen. Microbiol. 42, 403-410. doi: 10.1099/00221287-42-3-403

Becker, E. C., and Meyer, R. J. (2000). Recognition of oriT for DNA processing at termination of a round of conjugal transfer. J. Mol. Biol. 300, 1067-1077. doi: 10.1006/jmbi.2000.3902

Berriatua, E., Ziluaga, I., Miguel-Virto, C., Uribarren, P., Juste, R., Laevens, S., et al. (2001). Outbreak of subclinical mastitis in a flock of dairy sheep associated with Burkholderia cepacia complex infection. J. Clin. Microbiol. 39, 990-994. doi: 10.1128/JCM.39.3.990-994.2001

Chandler, M., and Galas, D. J. (1983). Cointegrate formation mediated by Tn9. II. Activity of IS1 is modulated by external DNA sequences. J. Mol. Biol. 170, 61-91. doi: 10.1016/S0022-2836(83)80227-7

\section{AUTHOR CONTRIBUTIONS}

EF contributed to the experimental set-up, analysis, interpretation of data and drafting the work; SB and MG contributed to the experimental results; DO and FS contributed to the conception of the work and critical revision of the manuscript; FS, AV, and ML contributed to the design of the work, interpretation of data, drafting, and revising the manuscript. All the authors approved the final version of the manuscript.

\section{FUNDING}

This work was supported by grants BIO2013-46414-P from the Spanish Ministry of Economy and Competitiveness to ML and BFU2011-25658 from the Spanish Ministry of Science and Innovation to FS. U1047 is supported by INSERM and the Université de Montpellier. AV was recipient of a "Chercheur d'avenir" award from La Region Languedoc-Roussillon, and SB was supported by a doctoral fellowship co-funded by the Region Languedoc-Roussillon and INSERM.

\section{ACKNOWLEDGMENTS}

We are grateful to Juan M. García-Lobo for assistance with the bioinformatics analysis.

\section{SUPPLEMENTARY MATERIAL}

The Supplementary Material for this article can be found online at: http://journal.frontiersin.org/article/10.3389/fmolb. 2016.00016

Cho, H., and Winans, S. C. (2007). TraA, TraC and TraD autorepress two divergent quorum-regulated promoters near the transfer origin of the $\mathrm{Ti}$ plasmid of Agrobacterium tumefaciens. Mol. Microbiol. 63, 1769-1782. doi: 10.1111/j.13652958.2007.05624.x

Christie, P. J., and Vogel, J. P. (2000). Bacterial type IV secretion: conjugation systems adapted to deliver effector molecules to host cells. Trends Microbiol. 8, 354-360. doi: 10.1016/S0966-842X(00)01792-3

Coenye, T., and Vandamme, P. (2003). Diversity and significance of Burkholderia species occupying diverse ecological niches. Environ. Microbiol. 5, 719-729. doi: 10.1046/j.1462-2920.2003.00471.x

Crameri, R., Davies, J. E., and Hütter, R. (1986). Plasmid curing and generation of mutations induced with ethidium bromide in streptomycetes. J. Gen. Microbiol. 132, 819-824. doi: 10.1099/00221287-132-3-819

Darling, P., Chan, M., Cox, A. D., and Sokol, P. A. (1998). Siderophore production by cystic fibrosis isolates of Burkholderia cepacia. Infect. Immun. 66, 874-877.

Demarre, G., Guérout, A. M., Matsumoto-Mashimo, C., Rowe-Magnus, D. A., Marlière, P., and Mazel, D. (2005). A new family of mobilizable suicide plasmids based on broad host range R388 plasmid (IncW) and RP4 plasmid $(\operatorname{IncP} \alpha)$ conjugative machineries and their cognate Escherichia coli host strains. Res. Microbiol. 156, 245-255. doi: 10.1016/j.resmic.2004. 09.007

Dennis, J. J., and Zylstra, G. J. (1998). Plasposons: modular self-cloning minitransposon derivatives for rapid genetic analysis of gram-negative bacterial genomes. Appl. Environ. Microbiol. 64, 2710-2715.

Engledow, A. S., Medrano, E. G., Mahenthiralingam, E., LiPuma, J. J., and Gonzalez, C. F. (2004). Involvement of a plasmid-encoded type IV secretion 
system in the plant tissue watersoaking phenotype of Burkholderia cenocepacia. J. Bacteriol. 186, 6015-6024. doi: 10.1128/JB.186.18.6015-6024.2004

Estrada-De Los Santos, P., Vinuesa, P., Martínez-Aguilar, L., Hirsch, A. M., and Caballero-Mellado, J. (2013). Phylogenetic analysis of burkholderia species by multilocus sequence analysis. Curr. Microbiol. 67, 51-60. doi: 10.1007/s00284013-0330-9

Fernández-González, E., de Paz, H. D., Alperi, A., Agúndez, L., Faustmann, M., Sangari, F. J., et al. (2011). Transfer of R388 derivatives by a pathogenesisassociated type IV secretion system into both bacteria and human cells. J. Bacteriol. 193, 6257-6265. doi: 10.1128/JB.05905-11

Fernández-López, C., Lorenzo-Díaz, F., Pérez-Luque, R., Rodríguez-González, L., Boer, R., Lurz, R., et al. (2013). Nicking activity of the pMV158 MobM relaxase on cognate and heterologous origins of transfer. Plasmid 70, 120-130. doi: 10.1016/j.plasmid.2013.03.004

Francia, M. V., Varsaki, A., Garcillán-Barcia, M. P., Latorre, A., Drainas, C., and De La Cruz, F. (2004). A classification scheme for mobilization regions of bacterial plasmids. FEMS Microbiol. Rev. 28, 79-100. doi: 10.1016/j.femsre.2003.09.001

Garcillán-Barcia, M. P., and de la Cruz, F. (2008). Why is entry exclusion an essential feature of conjugative plasmids? Plasmid 60, 1-18. doi: 10.1016/j.plasmid.2008.03.002

Garcillán-Barcia, M. P., Francia, M. V., and De La Cruz, F. (2009). The diversity of conjugative relaxases and its application in plasmid classification. FEMS Microbiol. Rev. 33, 657-687. doi: 10.1111/j.1574-6976.2009. 00168.x

Gonzalez, C. F., Pettit, E. A., Valadez, V. A., and Provin, E. M. (1997). Mobilization, cloning, and sequence determination of a plasmid-encoded polygalacturonase from a phytopathogenic Burkholderia (Pseudomonas) cepacia. Mol. Plant. Microbe Interact. 10, 840-851. doi: 10.1094/MPMI.1997.10.7.840

Goven, J. R. W., Brown, P. H., Maddison, J., Doherty, C. J., Nelson, J. W., Dodd, M., et al. (1993). Evidence for transmission of Pseudomonas cepacia by social contact in cystic fibrosis. Lancet 342, 15-19. doi: 10.1016/0140-6736(93) 91881-L

Grandoso, G., Avila, P., Cayón, A., Hernando, M. A., Llosa, M., and de la Cruz, F. (2000). Two active-site tyrosyl residues of protein TrwC act sequentially at the origin of transfer during plasmid R388 conjugation. J. Mol. Biol. 295, 1163-1172. doi: 10.1006/jmbi.1999.3425

Grant, S. G., Jessee, J., Bloom, F. R., and Hanahan, D. (1990). Differential plasmid rescue from transgenic mouse DNAs into Escherichia coli methylationrestriction mutants. Proc. Natl. Acad. Sci. U.S.A. 87, 4645-4649. doi: 10.1073/pnas.87.12.4645

Heidelberg, J. F., Eisen, J. A., Nelson, W. C., Clayton, R. A., Gwinn, M. L., Dodson, R. J., et al. (2000). DNA sequence of both chromosomes of the cholera pathogen Vibrio cholerae. Nature 406, 477-483. doi: 10.1038/35020000

Holden, M. T. G., Seth-Smith, H. M. B., Crossman, L. C., Sebaihia, M., Bentley, S. D., Cerdeño-Tárraga, A. M., et al. (2009). The genome of Burkholderia cenocepacia $\mathrm{J} 2315$, an epidemic pathogen of cystic fibrosis patients. J. Bacteriol. 91, 261-277. doi: 10.1128/JB.01230-08

Jandle, S., and Meyer, R. (2006). Stringent and relaxed recognition of oriT by related systems for plasmid mobilization: implications for horizontal gene transfer. J. Bacteriol. 188, 499-506. doi: 10.1128/JB.188.2.499-506.2006

Kelley, L. A., Mezulis, S., Yates, C. M., Wass, M. N., and Sternberg, M. J. E. (2015). The Phyre2 web portal for protein modeling, prediction and analysis. Nat. Protoc. 10, 845-858. doi: 10.1038/nprot.2015.053

Koichiro, T., Peterson, D., Peterson, N., Stecher, G., Nei, M., and Kumar, S. (2011). MEGA5: molecular evolutionary genetics analysis. Mol. Biol. Evol. 28, 2731-2739. doi: 10.1093/molbev/msr121

Kovach, M. E., Phillips, R. W., Elzer, P. H., Roop, R. M., and Peterson, K. M. (1994). pBBR1MCS: a broad-host-range cloning vector. Biotechniques 16, 800-802.

Langer, P. J., and Walker, G. C. (1981). Restriction endonuclease cleavage map of pKM101: relationship to parental plasmid R46. Mol. Gen. Genet. 182, 268-272. doi: $10.1007 / \mathrm{BF} 00269669$

Lawley, T. D., Klimke, W. A., Gubbins, M. J., and Frost, L. S. (2003). F factor conjugation is a true type IV secretion system. FEMS Microbiol. Lett. 224, 1-15. doi: 10.1016/S0378-1097(03)00430-0

LiPuma, J. J. (1998a). Burkholderia cepacia epidemiology and pathogenesis: implications for infection control. Curr. Opin. Pulm. Med. 4, 337-341.

LiPuma, J. J. (1998b). Burkholderia cepacia. Management issues and new insights. Clin. Chest Med. 19, 473-486, vi.
LiPuma, J. J. (2003). Burkholderia and emerging pathogens in Cystic Fibrosis. Semin. Respir. Crit. Care Med. 24, 681-692. doi: 10.1055/s-2004-815664

Llosa, M., Bolland, S., and de la Cruz, F. (1994). Genetic organization of the conjugal DNA processing region of the IncW plasmid R388. J. Mol. Biol. 235, 448-464. doi: 10.1006/jmbi.1994.1005

Llosa, M., Grandoso, G., Hernando, M. A., and de la Cruz, F. (1996). Functional domains in protein TrwC of plasmid R388: dissected DNA strand transferase and DNA helicase activities reconstitute protein function. J. Mol. Biol. 264, 56-67. doi: 10.1006/jmbi.1996.0623

Llosa, M., Zunzunegui, S., and de la Cruz, F. (2003). Conjugative coupling proteins interact with cognate and heterologous VirB10-like proteins while exhibiting specificity for cognate relaxosomes. Proc. Natl. Acad. Sci. U.S.A. 100, 10465-10470. doi: 10.1073/pnas. 1830264100

Lucas, M., González-Pérez, B., Cabezas, M., Moncalian, G., Rivas, G., and De La Cruz, F. (2010). Relaxase DNA binding and cleavage are two distinguishable steps in conjugative DNA processing that involve different sequence elements of the nic site. J. Biol. Chem. 285, 8918-8926. doi: 10.1074/jbc.M109.057539

Mahenthiralingam, E., Coenye, T., Chung, J. W., Speert, D. P., Govan, J. R. W., Taylor, P., et al. (2000). Diagnostically and experimentally useful panel of strains from the Burkholderia cepacia complex. J. Clin. Microbiol. 38, 910-913.

Martinez, E., and de la Cruz, F. (1988). Transposon Tn21 encodes a RecAindependent site-specific integration system. Mol. Gen. Genet. 211, 320-325. doi: 10.1007/BF00330610

Matson, S. (1990). DNA Helicases. Annu. Rev. Biochem. 59, 289-329. doi: 10.1146/annurev.bi.59.070190.001445

Moncalián, G., and De La Cruz, F. (2004). DNA binding properties of protein TrwA, a possible structural variant of the Arc repressor superfamily. Biochim. Biophys. Acta 1701, 15-23. doi: 10.1016/j.bbapap.2004. 05.009

Moncalián, G., Grandoso, G., Llosa, M., and de la Cruz, F. (1997). oriT-processing and regulatory roles of TrwA protein in plasmid R388 conjugation. J. Mol. Biol. 270, 188-200. doi: 10.1006/jmbi.1997.1082

O’Brien, F. G., Eto, K. Y., Murphy, R. J. T., Fairhurst, H. M., Coombs, G. W., Grubb, W. B., et al. (2015). Origin-of-transfer sequences facilitate mobilisation of nonconjugative antimicrobial-resistance plasmids in Staphylococcus aureus. Nucleic Acids Res. 43, 7971-7983. doi: 10.1093/nar/gkv755

Overbeek, R., Olson, R., Pusch, G. D., Olsen, G. J., Davis, J. J., Disz, T., et al. (2014). The SEED and the Rapid Annotation of microbial genomes using Subsystems Technology (RAST). Nucleic Acids Res. 42, D206-D214. doi: 10.1093/nar/gkt1226

Pérez-Mendoza, D., Lucas, M., Muñoz, S., Herrera-Cervera, J. A., Olivares, J., De La Cruz, F., et al. (2006). The relaxase of the Rhizobium etli symbiotic plasmid shows nic site cis-acting preference. J. Bacteriol. 188, 7488-7499. doi: 10.1128/JB.00701-06

Pollet, R. M., Ingle, J. D., Hymes, J. P., Eakes, T. C., Eto, K. Y., Kwong, S. M., et al. (2016). Processing of non-conjugative resistance plasmids by conjugation nicking enzyme of Staphylococci. J. Bacteriol. 198, 888-897. doi: 10.1128/JB.00832-15

Ramage, H. R., Connolly, L. E., and Cox, J. S. (2009). Comprehensive functional analysis of Mycobacterium tuberculosis toxin-antitoxin systems: Implications for pathogenesis, stress responses, and evolution. PLoS Genet. 5:e1000767. doi: 10.1371/journal.pgen.1000767

Sadler, J. R., Tecklenburg, M., and Betz, J. L. (1980). Plasmids containing many tandem copies of a synthetic lactose operator. Gene 8, 279-300. doi: 10.1016/0378-1119(80)90005-0

Sajjan, S. U., Carmody, L. A., Gonzalez, C. F., and Lipuma, J. J. (2008). A type IV secretion system contributes to intracellular survival and replication of Burkholderia cenocepacia. Infect. Immun. 76, 5447-5455. doi: 10.1128/IAI.0 0451-08

Sambrook, J. W., and Russell, D. (2001). Molecular cloning: A Laboratory Manual. Cold Spring Harbor, NY: Cold Spring Harbor Laboratory Press.

Sepúlveda, E., Pérez-Mendoza, D., Ramírez-Romero, M. A., Soto, M. J., López-Lara, I. M., Geiger, O., et al. (2008). Transcriptional interference and repression modulate the conjugative ability of the symbiotic plasmid of Rhizobium etli. J. Bacteriol. 190, 4189-4197. doi: 10.1128/JB.0 0041-08

Speert, D. P. (2002). Advances in Burkholderia cepacia complex. Paediatr. Respir. Rev. 3, 230-235. doi: 10.1016/S1526-0542(02)00185-9 
Valvano, M. A. (2015). Intracellular survival of Burkholderia cepacia complex in phagocytic cells. Can. J. Microbiol. 615, 607-615. doi: 10.1139/cjm-2015-0316

Vandamme, P., and Dawyndt, P. (2011). Classification and identification of the Burkholderia cepacia complex: past, present and future. Syst. Appl. Microbiol. 34, 87-95. doi: 10.1016/j.syapm.2010.10.002

Varga, J. J., Losada, L., Zelazny, A. M., Kim, M., McCorrison, J., Brinkac, L., et al. (2013). Draft Genome Sequences of Burkholderia cenocepacia ET12 Lineage Strains K56-2 and BC7. Genome Announc. 1, e00841-e00813. doi: 10.1128/genomeA.00841-13

Wernegreen, J. J., and Riley, M. A. (1999). Comparison of the evolutionary dynamics of symbiotic and housekeeping loci: a case for the genetic coherence of rhizobial lineages. Mol. Biol. Evol. 16, 98-113. doi: 10.1093/oxfordjournals.molbev.a026041

Williams, S. L., and Schildbach, J. F. (2006). Examination of an inverted repeat within the $\mathrm{F}$ factor origin of transfer: context dependence of $\mathrm{F}$ TraI relaxase DNA specificity. Nucleic Acids Res. 34, 426-435. doi: 10.1093/nar/ gkj444

Williams, S. L., and Schildbach, J. F. (2007). TraY and integration host factor oriT binding sites and $\mathrm{F}$ conjugal transfer: sequence variations, but not altered spacing, are tolerated. J. Bacteriol. 189, 3813-3823. doi: 10.1128/JB. 01783-06

Wong, J. J. W., Lu, J., Edwards, R. A., Frost, L. S., and Glover, J. N. M. (2011). Structural basis of cooperative DNA recognition by the plasmid conjugation factor, TraM. Nucleic Acids Res. 39, 6775-6788. doi: 10.1093/nar/gkr296
Yamaguchi, Y., Park, J.-H., and Inouye, M. (2011). Toxin-Antitoxin systems in bacteria and Archaea. Annu. Rev. Genet. 45, 61-79. doi: 10.1146/annurevgenet-110410-132412

Zechner, E. L., La Cruz, F., Eisenbrandt, R., Grahn, A. M., Koraimann, G., Lanka, E., et al. (2000). "Conjugative-DNA transfer processes," in The Horizontal Gene Pool, ed C. Thomas (Reading: Harwood Academic Publisher), 87-174.

Zhang, R., LiPuma, J. J., and Gonzalez, C. F. (2009). Two type IV secretion systems with different functions in Burkholderia cenocepacia K56-2. Microbiology 155, 4005-4013. doi: 10.1099/mic.0.033043-0

Zhong, Z., Helinski, D., and Toukdarian, A. (2005). Plasmid host-range: restrictions to $\mathrm{F}$ replication in Pseudomonas. Plasmid 54, 48-56. doi: 10.1016/j.plasmid.2004.11.001

Conflict of Interest Statement: The authors declare that the research was conducted in the absence of any commercial or financial relationships that could be construed as a potential conflict of interest.

Copyright (๑) 2016 Fernández-González, Bakioui, Gomes, O'Callaghan, Vergunst, Sangari and Llosa. This is an open-access article distributed under the terms of the Creative Commons Attribution License (CC BY). The use, distribution or reproduction in other forums is permitted, provided the original author(s) or licensor are credited and that the original publication in this journal is cited, in accordance with accepted academic practice. No use, distribution or reproduction is permitted which does not comply with these terms. 